\title{
Single Chip Potentiostat Measurement System
}

\author{
Pavel Steffan, Radimir Vrba \\ Brno University of Technology, Dept. of Microelectronics \\ Udolni 53, CZ-60200 Brno, Czech Republic \\ $\{$ steffan,vrba\}@feec.vutbr.cz
}

\begin{abstract}
The paper describes new approach of potentiostat for very low sensor current measurements. Recent potentiostats were used as measurement devices which were standalone and sensors were connected by a screened wire. These concepts are inconvenient for the presented very low current measurements. The new approach is based on the potentiostat circuitry integrated on a chip that should be directly contacted to a Thick Film Technology (TFT) sensor.
\end{abstract}

\section{Introduction}

The new approach of the amperometric sensor measurement is given. It solves the problem of measurement of low currents in order of $\mathrm{nA}$ for electrochemical biosensors. However the sensitivity of integrated measurement system, which processes biosens or output currents, has to be in order of pA. There are leakage currents and noise constraints which could be avoided by on-chip solution. The onchip solution allows direct chip connection on the bas ic ceramic plate of biosensors. It means the idea is similar to hybrid integrated circuit. Main advantage of this approach is keeping sensitive low potential and low current ASIC electronic circuitry placed just on the TFT sensor apart of control and supply electronics of portable device. Additional advantages of the sensors fabricated using TFT are low cost, small dimensions, good reproducibility, chemical, mechanical and electrical properties of electrodes and well accessible and ecological fabrication process.

The electrochemical applications are widespread. The preparation of micros ensor brings issues of large instrumentation connected with long leads. This set is sensitive to noise induced into measuring path. It is very important to min imize the measuring path to decrease noise because of very small current and voltage measured on microsensor. The potentiostat design and construction is discussed from the era of electron tubes discovery. Modem techniques and problems of design with modern circuitry as operating amplifiers are well described e.g. in [1], [2]. Then the requirements to builtup portable instrumentation brought a need to minimize power consumption [3]. Modem design utilizes CMOS technology for microchip fabrication [4]. The very simple potentiostat was integrated in this work where circuitry was presented with basic three operating amplifiers [5], or additionally supplemented with digital part for signal pre-processing [4], working at narrow range of measured currents .

To reach the highest possible sensitivity of the potentiostat, there was necessary to create a new approach of measurements. To minimize noise and other parasitic

Please use the following format when citing this chapter:

Steffan, P., Vrba, R., 2007, in IFIP International Federation for Information Processing, Volume 245, Personal Wireless Communications, eds. Simak, B., Bestak, R., Kozowska, E., (Boston: Springer), pp. 653-659. 
parameters, the integrated potentiostat placed directly on the body of sensor (see Fig. 4) was developed, designed and manufactured.

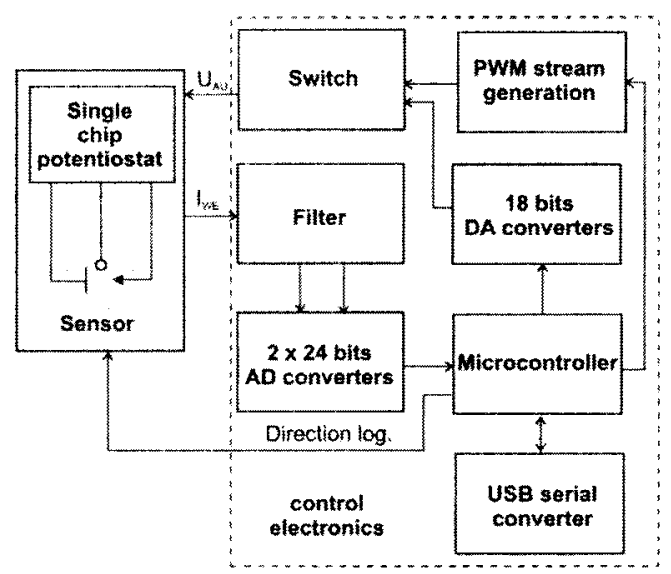

Fig. 1. New approach of potentiostat

The output sensor signals reflect current and potential of the working electrode. The potential is controlled by a programmable triangle voltage source. The required period of this signal supplying triangle is switchable in the range of $2 \mathrm{~s}$ to $60 \mathrm{~min}$. A high linearity of the output signal is a condition for a precise measurement. The previous system solved this problem either by using fully analog integrator [6] (it had problems with large linear capacitor, period switching, small currents) or by counters and DA converters with filtered output (problems with linearity, not suitable for on chip systems). Our novel solution of the triangle voltage source is based on the generation of a digital PWM (Pulse Wide Modulation) stream, which means that the voltage is equal to the requested output voltage and following low pass filtration. The mean value of the stream must be accurately defined in time with the triangle shape.

\section{Sensor}

The sensor is located on a corundum ceramic base. On th is surface the working, the reference and the auxiliary electrodes are applied. The working and the auxiliary electrodes are made of gold and the reference one is made of silver in the standard product. At the end of the sensor there is a contacting field which is connected with the active part by the silver conducting paths covered by a dielectric protection layer. The solution of the issues during measure ments is to miniaturize the circuitry of the instrumentation. Taking measurements by potentiometric method can be done only with potentiostat which is the most important circuitry in electrochemical analys is. The potentiostat should work at wide dynamic range of 8 orders; it means the current ranges $10 \mathrm{pA}$ to $1 \mathrm{~mA}$. The total current range has to be dividend into partial ranges because very small output voltage of converter current/voltage is reached for the smallest current. The current subranges changing keep good $\mathrm{S} / \mathrm{N}$ ration within total 
range. The subranges are adjusted by controlled resistances in feed-back loop. The measurement of current has to automatically change the subrange according to the value of the output voltage. The subrange control should not make high glitches to measured signal. Glitches can cause significant error in measurement because of influencing electrode reaction.

The mic ropotentios tat was designed with circuits shown in simplified Fig. 6. All parts were integrated together with digital part for subrange control and correction constant stored in PROM with 48 bits (see topology in Fig. 2). The me mory is downloaded by top system which is providing and controlling the measuring method to know the system parameters which are formed by mic ropotentios tat and electrode system and to do an automatic correction of measured signal. The microchip of the micropotentiostat is presented in Fig. 6. The microchip is mounted on thick-film sensor and connected by aluminu m wire bonding (see Fig. 3 and 4).

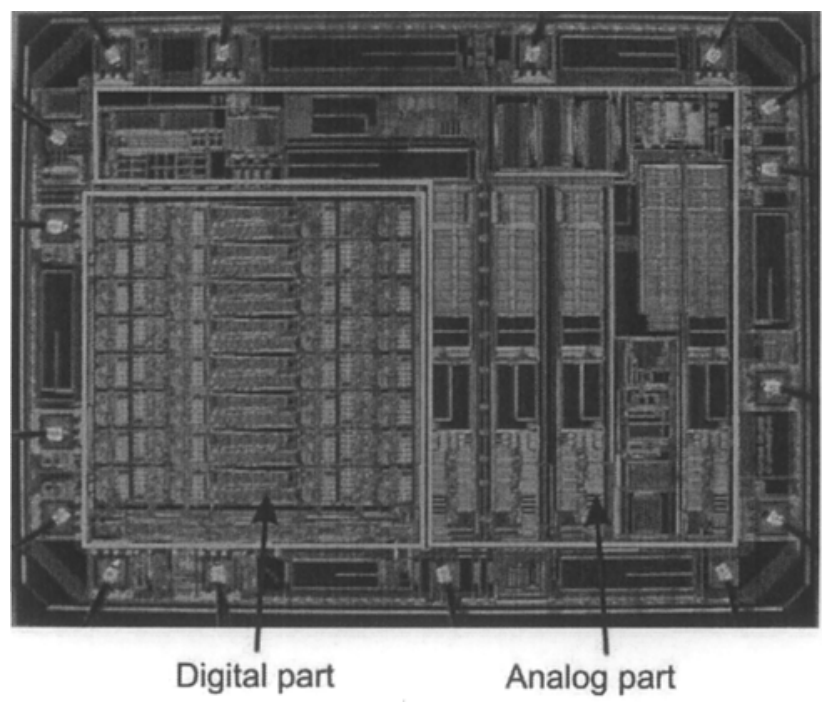

Fig. 2. Topology of designed microchip

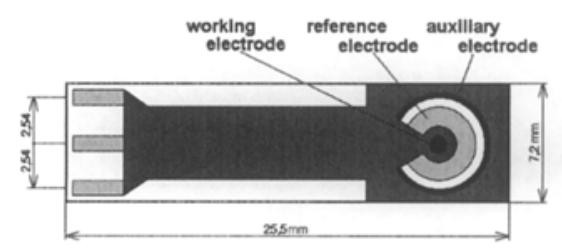

Fig. 3. Standard sensor

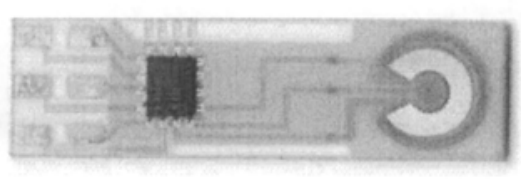

Fig. 4. Modified sensor 


\section{Chip Layout}

Chip was designed in technology AMIS CMOS 0,7 $\mu \mathrm{m}$. The integrated measurement system has two sections. Analog section is designed to control the potential on RE and to measure the current which flows through the sensor. This part is very important to settle final accuracy of measurement because this system measures very low currents in subranges from $1 \mathrm{nA}$ to $1 \mathrm{~mA}$. The measured signal is amplified and converted into current (Fig. 5,6). Th is signal features high absolute resistance against electric and magnetic interference. The simplified circuitry of the analog part is presented in Fig 6 . The chip is powered by $5 \mathrm{~V} \mathrm{DC}$. The internal analog ground potential is at $+2,5 \mathrm{~V}$ level related to external analog ground, i.e. the chip internal supply span is $\pm 2,5 \mathrm{~V}$ to internal ana log ground.

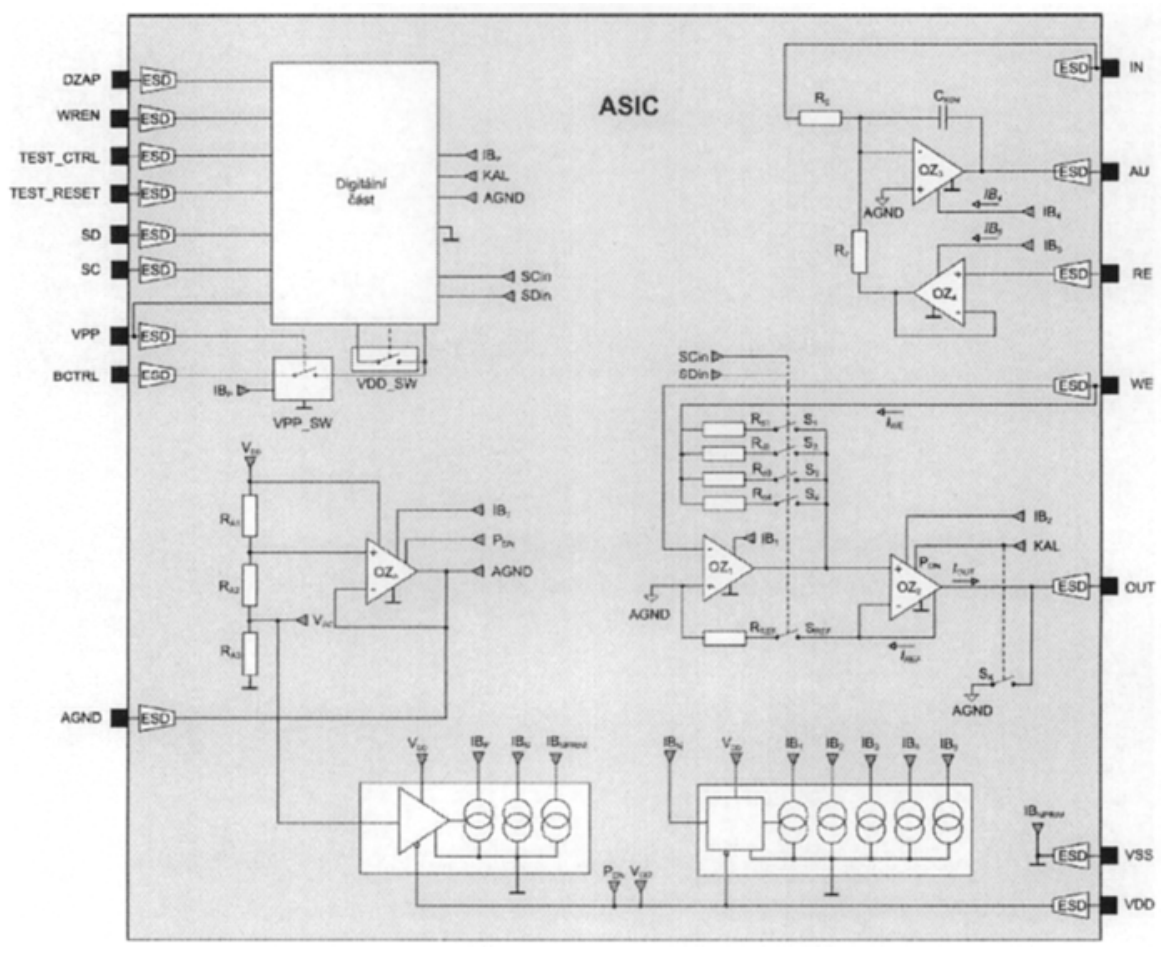

Fig. 5. The chip block diagram

Parameters of the type and series of the sensor are stored in the digital part of the chip. This part includes PROM memory, control logic, multiplexers and shift registers. 


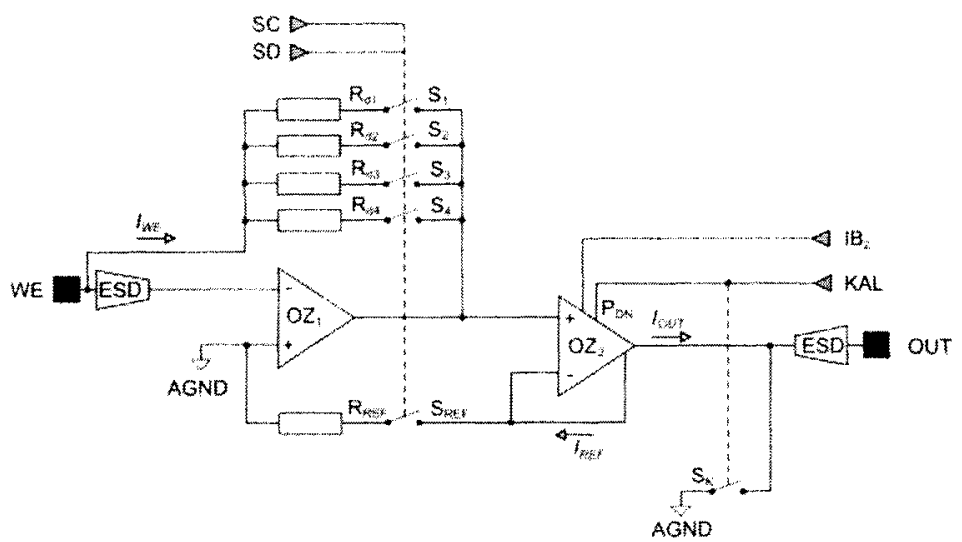

Fig. 6. Simplified circu it of analog part

\section{Results}

After the measurement system was properly checked (see Fig. 10) and tested, it was used for sensor measuring by the cyclic voltampere method. The intelligent measuring sensor was plunged into the $10 \mathrm{ml}$ of $10 \mathrm{mM}$ ferro-ferrikyanidu liquid. The results of the measuring are drawn in Fig. 11. These results were compared with measurement results of professional analyzer Sycopel AEW 2-10. Correct function of the proposed ASIC was proved.

\section{Relative linear error}

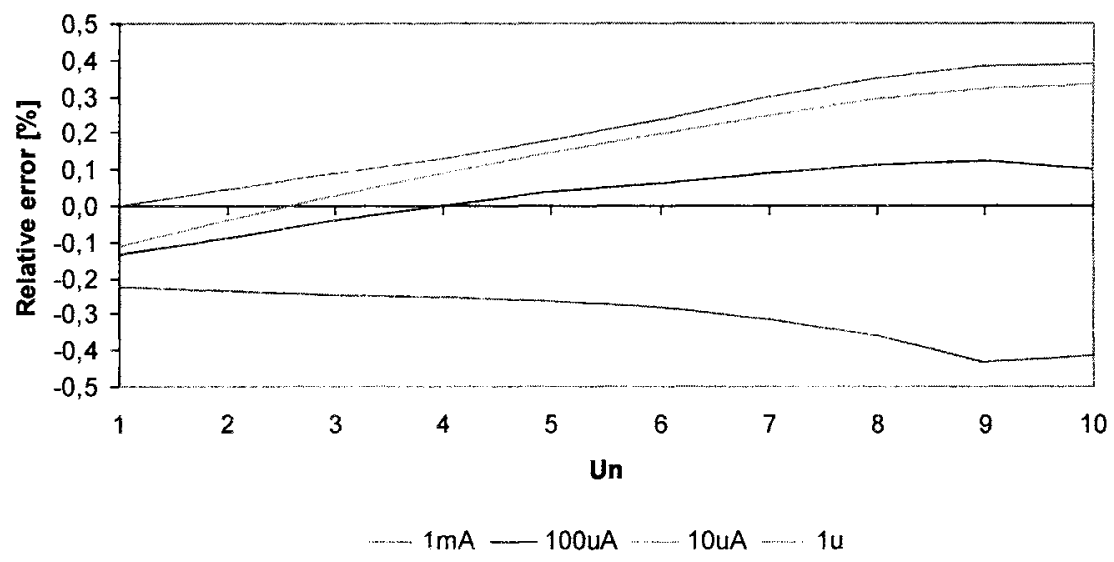

Fig. 7. Relative lin earity error 


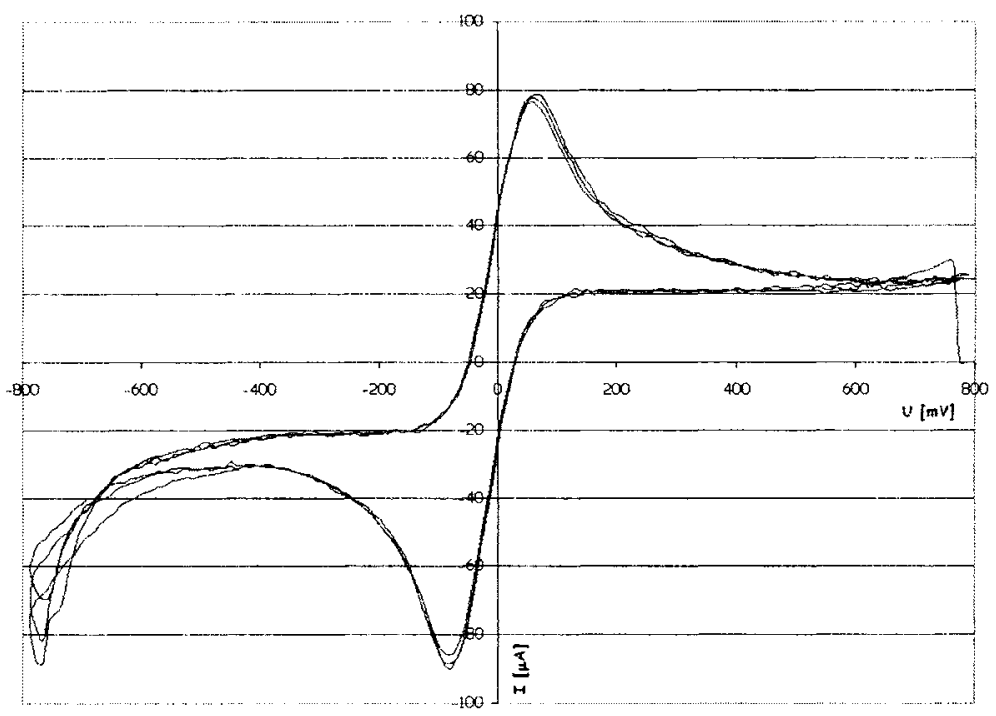

Fig. 8. The resulting plot of measured data

\section{Conclusions}

The new approach of the amperometric sensor measurement is given. It solves the problem of measurement of low currents (in order of $\mathrm{nA}$ ) from electrochemical biosensors. However the sensitivity of integrated measurement system, which process output currents from biosensor, has to be in order of $\mathrm{pA}$. There are leakage currents and noise constraints which could be avoided by on-chip solution. The on-chip approach allows direct chip connection on the basic ceramic plate of biosensors. It means the idea is similar to hybrid integrated circuit. Main advantage of this solution is separating sensitive low potential and low current ASIC electronic circuitry placed just on the TFT sensor apart of control and supply electronics of portable device. Additional advantages of the sensors fabricated using TFT are low cost, small dimensions, good reproducibility, chemical, mechanical and electrical properties of electrodes and well accessible and ecological fabrication process.

This paper focuses to deal with the design of a mixed signal ASIC single chip measurement system for embedded digital sensing of TFT biosensors. This ASIC monolithic measurement system is embedded on thick film amperometric sensors. The sensor applies fused alumina substrate processed by thick film technology. The ASIC prototype prepared in CMOS $0.7 \mu \mathrm{m}$ technology contains mixed signal analog measurement block and programmable digital calibration PROM memory located on the same chip. The digital part of the chip of the smart sensor contains 48 OTP (One Time Programmable) cells that are supposed to store calibration data for the precision measurement. The full system is controlled by embedded software. PC and 
programmer communicate with the biosensor embedded ASIC chip via a USB interface.

Acknowedgment. Funding for this work was provided by the Ministry of Industry and Commerce under the contract FT-TA/050 and Czech Ministry of Education in the frame of Research Program MSM0021630503 MIKROSYN New Trends in Microelectronic Systems and Nanotechnologies.

\section{References}

1. Potentiostats. Bank Elektronic - Inteligent controls $\mathrm{GmbH}$, Clausthal - Zellerfeld

2. C. E. D. Chidsey, Lab B. Amperometry: Electrochemistry with a 3-Electrode Potentiostat. Chem 174: Instrumental and Physical Principles of Chemical Measurements, (2002), pp. 1-17.

3. Richard J. Reay, Anthony F. Flannery, Christopher W. Storment, Matthew D. Steinberg Christopher R. Lowe, A micropower amperometric potentiosta. Sensors and Actuators B 97 (2004), pp.284-289.

7. Michael Bollerott, Low Power Single-Chip CMOS Potentiostat. Fraunhofer Institut Mikroelektronische Schaltungen und Systeme, Duisburg, http://www.ims.fhgde, (1999)

5. Samuel P. Kounaves, Gregory T.A. Kovacs, Microfabricated electrochemical analysis system for heavy metal detection. Sensors and Actuators B 34 (1996), pp.450- 455.

6. Prokop, R. - Vrba, R. - Skocdopole, M. - Fujcik, L.: A Slow Triangle Generator for Smart Sensors, EDS (2003), ISBN 80-214-2452-4 